\title{
Pale vacuolated epithelial cells in epididymis of aflatoxin-treated mice
}

\author{
V. F. Agnes ${ }^{\dagger}$ and M. A. Akbarsha* \\ Department of Animal Science, School of Life Sciences, Bharathidhasan University, \\ Tiruchirappalli-620024, India
}

The responses of the mouse epididymal epithelium to subchronic doses of aflatoxin B1 were investigated in a histological study. Either few and large or small and profuse vacuoles containing an amorphous to dense periodic acid-Schiff-positive material were observed in the epithelium of all the segments of the epididymis. Resinembedded semi-thin sections and transmission electron microscopy indicated that these vacuoles were intracellular. The cells that contained these vacuoles were quite different in organization and electron density from the cell types already established in the epididymal epithelium and are designated as pale vacuolated epithelial cells. Owing to aflatoxin B1 toxicity, the apical membrane of some of the principal cells, either individually or in groups, disintegrated so that the principal cells released their contents into the lumen of the duct through development of a 'fistula'. Spermatozoa from the ductal lumen entered the principal cell fistula and reached the basal lamina. If extravasation of the spermatozoa via this route occurred, it would bring about an autoimmune response, leading to the formation of spermatic granulomas and the generation of anti-sperm antibodies. Extravasation of spermatozoa seems to be offset by the underlying basal cell, which is presumed to develop into a pale vacuolated epithelial cell to enclose the disintegrating principal cells and the spermatozoa arriving at the principal cell. Thus, the development of pale vacuolated epithelial cells may be a protective device preventing an autoimmune response to sperm antigens in the context of toxicant-induced degeneration of the principal cells of the epididymal epithelium.

\section{Introduction}

There has been renewed interest in male reproductive toxicology in view of the reported deleterious effects of drugs, environmental chemicals and industrial chemicals on human male reproductive structure and function (Sharpe, 1993). The possibility that such effects lead to male infertility also has implications in animal husbandry (Hafez et al., 1982; Pier, 1992) and poultry farming (Klein et al., 2000; Oliveira et al., 2000). The principal target organ of these toxicants in causing male reproductive toxicity is the testis, and various aspects of spermatogenesis and androgen biosynthesis are affected (Chapin et al., 1997; Kelce, 1997; Richburg, 2000). However, in addition to the testis, the epididymis may also be a target for the action of reproductive toxicants (Trasler et al., 1988; Akbarsha and Averal, 1996, 1998, 1999a,b; Klinefelter, 1997; Hess, 1998; Akbarsha and Sivasamy, 1998; Akbarsha et al., 2000). Epididymal toxicity gains importance in view of the role of the epididymis in post-testicular maturation of spermatozoa (Hinton and Palladino, 1995; Robaire and Viger, 1995; Cooper, 1995, 1998).

\footnotetext{
*Correspondence
}

Email: akbar@bdu.ernet.in

†Present address: Post-Graduate Department of Zoology, Holy Cross College, Tiruchirappalli-620002, India
Toxins from dietary sources can contribute to male reproductive toxicity, although this aspect is poorly studied. One of the well-known and widely prevalent groups of foodborne toxins is the aflatoxins. Aflatoxins are low molecular weight secondary metabolites produced by the fungi Aspergillus flavus and $A$. parasiticus, which contaminate various feeds and food commodities. There is considerable evidence for the carcinogenicity (Massey et al., 1995; Wogan, 1999) and mutagenicity (Buss et al., 1990; Choy, 1993; Wang and Groopman, 1999) of aflatoxins. Of the various food-borne aflatoxins, such as B1, B2, G1 and G2, aflatoxin B1 (AFB1) is the most implicated in hepatotoxicity (Buss et al., 1990; Wang and Groopman, 1999). In the liver, AFB1 exerts its mutagenic and carcinogenic effect via its metabolic activation by the cytochrome P450 enzymes to the toxic intermediate AFB1-epoxides. Some of these nucleophilic intermediates (for example, AFB1 8,9-epoxide) react with DNA, forming persistent adducts that can induce mutations. Other nucleophilic intermediates (for example, 8,9-dihydro-8, 9 dihydroxy-AFB1) may bind to cellular proteins, inducing cellular injury and eventual cell death (Fink-Gremmels, 1999). There is a paucity of information on the effects of AFB1 on the structure and function of the male reproductive system. The studies of Egbunike (1982, 1985) and Egbunike et al. (1980) indicate testicular degeneration, sloughing of germ cells and concomitant reduction in the rate and efficiency of sperm production in rats treated with 


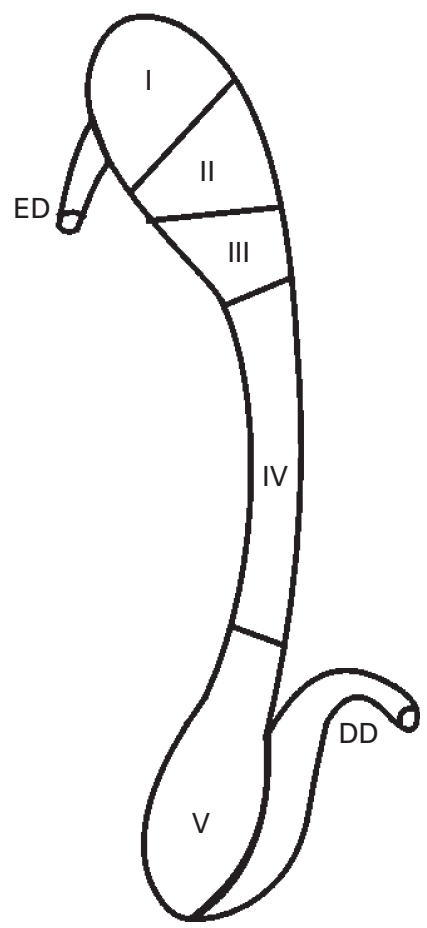

Fig. 1. Mouse epididymis showing segments I-V. ED, efferent duct; DD, deferent duct.

sub-lethal doses of AFB1. Hafez et al. (1982) reported that aflatoxins affect sperm counts and morphology in buffalo bulls. Sotomayor et al. (1999) investigated the action of AFB1 on DNA of testis and the consequent germ cell mutagenesis. However, little is known about the extent of the damaging effect of aflatoxins on the male reproductive tract, particularly the epididymis. Therefore, the aim of the present study was to investigate the response of the epididymis of adult mice to AFB1 treatment. Among the several changes, an extensive development of pale vacuolated epithelial cells (PVECs) was observed in all segments of the epididymis. The present study reports the occurrence of PVEC in the epithelium of the mouse epididymis, as a response to AFB1 treatment, and proposes its role and a tentative sequence of events leading to the origin of this cell type.

\section{Materials and Methods}

\section{Animals}

Male Swiss albino mice, 90 days old, raised from a stock obtained from Frederick Institute of Plant Protection and Toxicology (FIPPAT, Padappai, Chennai) and housed in individual cages with bedding, were used in the experiments. The mice were fed with standard pellet feed (Lipton India Ltd, Bangalore) and tap water ad libitum. All experimental protocols were approved by the local ethics community.

\section{Experimental protocol}

AFB1 (Sigma, St Louis, MO) was dissolved in corn oil and ethanol (95:5) and administered to the mice by i.p. injection. Control mice received vehicle alone. A biologically active dose of 50-60 $\mathrm{g} \mathrm{kg}^{-1}$ body weight for 4 weeks in rats was reported by Egbunike et al. (1980). Before the present study, AFB1 was tested in doses varying from 50 to $250 \mu \mathrm{g} \mathrm{kg}^{-1}$ day $^{-1}$ for 15 days (five mice in each of the experimental and control groups) to find the dose-response. A daily dose of $50 \mu \mathrm{g} \mathrm{kg}{ }^{-1}$ day $^{-1}$ was found to be sufficient to bring about histopathological changes in the male reproductive system and this dose was used throughout the present study. Aflatoxin was administered at $50 \mathrm{gg} \mathrm{kg}^{-1}$ day $^{-1}$ to the mice for $7,15,35$ and 45 days (ten mice in each of the experimental and control groups). At the end of the respective experimental periods, the epididymides of five mice in each group were excised and fixed in Bouin's fluid, embedded in paraffin wax and $5 \mu \mathrm{m}$ serial sections were cut and stained in Harris's haematoxylin and eosin or periodic acid-Schiff (PAS) and haematoxylin for observation under a light microscope. Micrometric measurement of the diameter of the vacuoles in the epithelium was made (ten vacuoles were selected at random in each of the epididymal segments of each mouse) using a calibrated ocular micrometer, and the data were used to calculate means and standard deviations.

The reproductive systems of the remaining five mice in each group were perfused with Karnovsky's (1965) fluid and thin slices of the epididymal segments I-V (initial segment, intermediate zone, head, body and tail) defined by Abe et al. (1982) (Fig. 1) were immersion-fixed in $2.5 \%(\mathrm{w} / \mathrm{v})$ glutaraldehyde and post-fixed in 1\% (w/v) osmium tetroxide. The perfusion fixation method adopted was that of Hess and Moore (1993) which uses a peristaltic pump to allow a steady flow of physiological saline $(0.9 \% \mathrm{NaCl})$ followed by the perfusate. Tissues were embedded in thin viscosity resin (Sigma). Semi-thin $1 \mu \mathrm{m}$ sections were obtained in a Reichert Jung ultratome and stained in toluidine blue-O for observation under a light microscope. Ultrathin sections were cut in a Leica ultratome, stained in uranyl acetate and lead citrate and observed under a Phillips 201C transmission electron microscope.

\section{Results}

\section{Observations under a light microscope: paraffin wax sections}

The epididymis of control mice did not contain vacuoles in the epithelium of any of the segments of the epididymis, but in the AFB1-treated mice vacuoles were always present. The vacuoles were more abundant in mice treated for 35 and 45 days. In segments I-IV, vacuoles were large but few (Fig. 2a-d) and, in segment $\mathrm{V}$, vacuoles were small but profuse (Fig. 2e). The lumen of the vacuoles contained an amorphous to dense PAS-positive material (Figs 2b and 3a). Occasionally, two or more vacuoles were in continuity or were separated by only a thin rim of cytoplasm (Fig. 3b). 

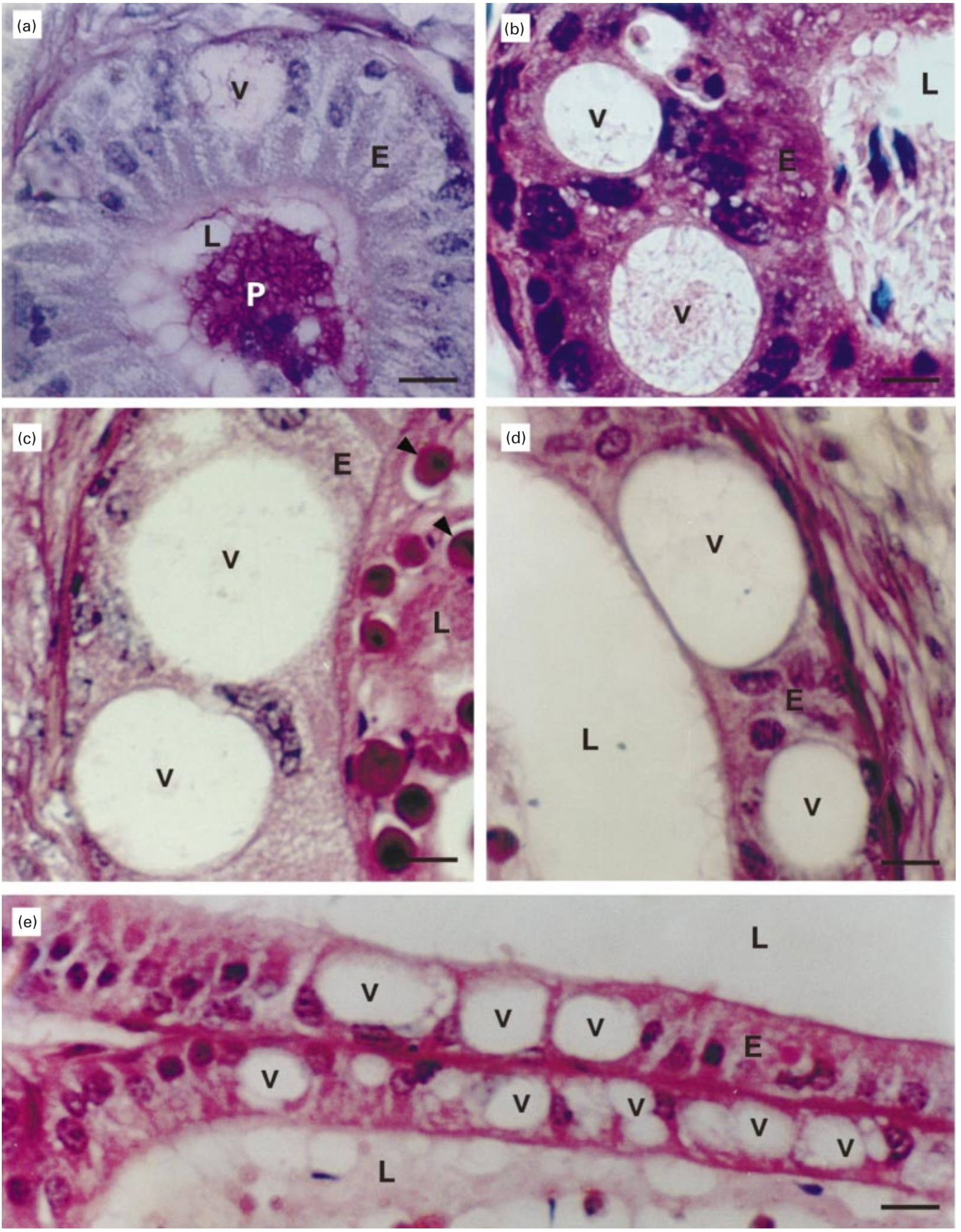

Fig. 2. Periodic acid-Schiff (PAS) and haematoxylin-stained paraffin wax sections showing vacuoles (V) in the epididymal epithelium (E) of Swiss mice treated with aflatoxin B1. (a) Segment I (initial segment); (b) segment II (intermediate zone); (c) segment III (caput) (arrowheads indicate cells that have arrived from the testis); (d) segment IV (corpus); (e) segment V (cauda). L, ductal lumen; P, PAS-positive material. Scale bars represent $15 \mu \mathrm{m}$. 

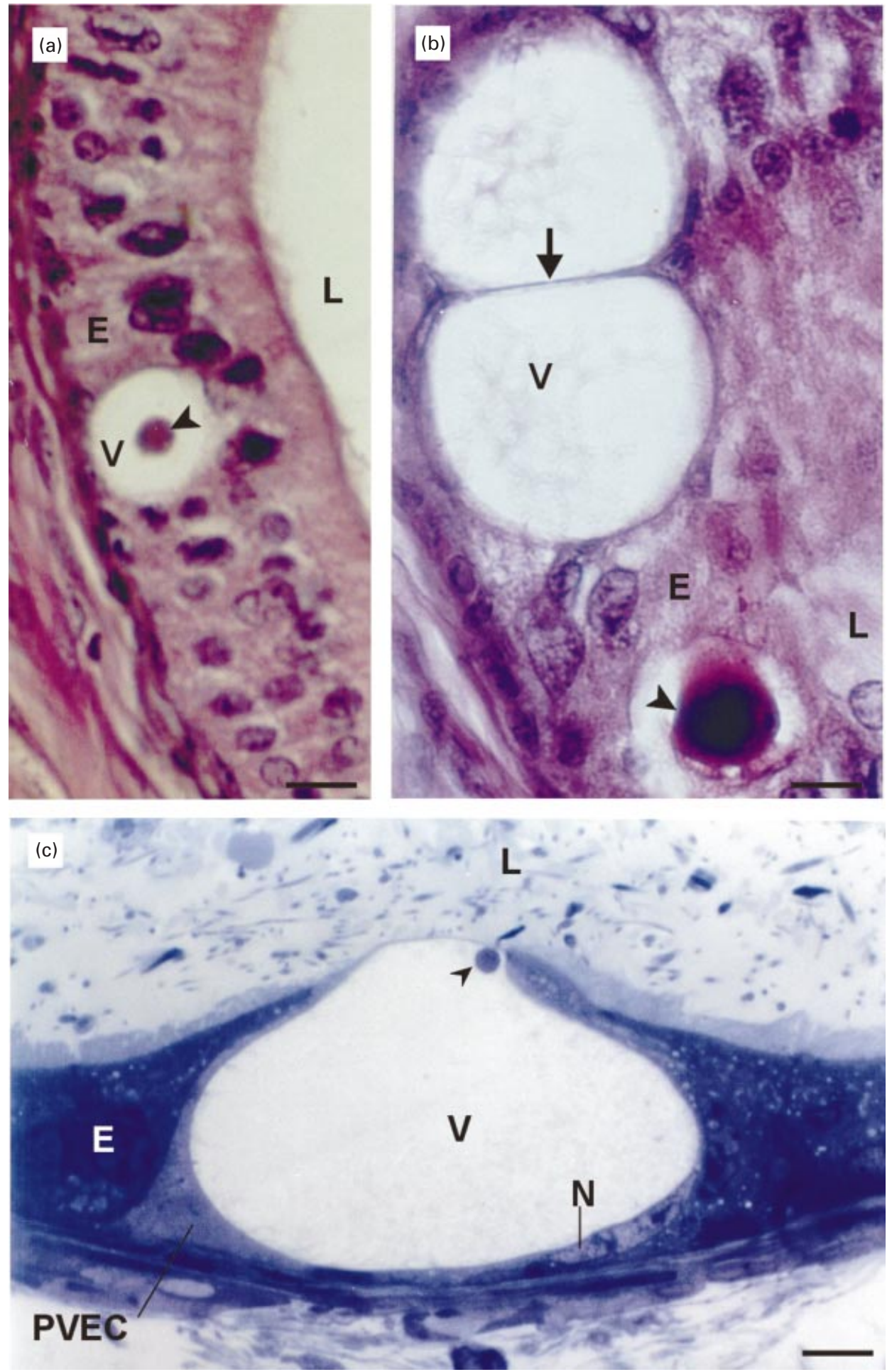

Fig. 3. (a) Haematoxylin and eosin-stained paraffin wax section of the epithelium (E) of segment IV of a mouse epididymis showing a highly condensed material (arrowhead) in the vacuole (V). (b) Periodic acid-Schiff (PAS) and haematoxylin-stained paraffin wax section showing two vacuoles $(\mathrm{V})$ in the epithelium (E) of segment I separated by a thin rim of cytoplasm (arrow); note the cyst enclosing the dense material (arrowhead). (c) Toluidine blue-O-stained semi-thin section showing a large vacuole in the epithelium (E) of segment $\mathrm{V}$; a pale vacuolated epithelial cell (PVEC) encloses the vacuole $(\mathrm{V})$, the content of which is partly amorphous and partly dense (arrowhead). $\mathrm{N}$, nucleus of the PVEC; L, ductal lumen. Scale bars represent $15 \mu \mathrm{m}(\mathrm{a}), 6.5 \mu \mathrm{m}$ (b) and $10 \mu \mathrm{m}$ (c). 


\section{Observations under a light microscope: semi-thin sections}

Semi-thin sections provided an indication that the entire vacuole in a transverse section was contained within a cell in which the nucleus was found in an eccentric location and the bulk of the cytoplasm was found as a thin rim encircling the vacuole (Fig. 3c). There were also indications of sparse microvilli extending from the cytoplasmic rim into the lumen over a short distance. The vacuole contained an amorphous to dense material. The cytoplasm of this cell stained lighter than the neighbouring principal cells.

\section{Ultrastructural observations}

The intracellular nature of the vacuole was confirmed by ultrastructural analysis (Fig. 4a). The cytoplasm of the vacuolated epithelial cell was less electron-dense than that of neighbouring principal cells. Therefore, these vacuolated epithelial cells were designated pale vacuolated epithelial cells (PVECs). Occasionally, a single PVEC contained two or more large or several small vacuoles. The heterochromatic and pleiomorphic nucleus was observed in various locations around the vacuole, but most often in an eccentric basal position. The cytoplasm of the PVEC contained smooth and rough endoplasmic reticulum around the nucleus and mitochondria were abundant. The cytoplasmic ring abounded with endosomes and multivesicular bodies. Short microvilli arose from the cytoplasmic rim and extended into the vacuole. The principal cells around the PVEC enveloped it in such a way that the apical ends of the principal cells came to lie on the top of the PVEC (Fig. 4a,b). The part of the principal cells that covered the top of the PVEC possessed apical zonulae occludentes (Fig. 4b).

\section{Origin of the pale vacuolated epithelial cell}

The following observations make a tentative sequence of events in the establishment of the PVECs. In the AFB1treated mice, some of the principal cells in all the segments appeared to undergo degenerative changes commencing with hyalination of the cytoplasm, and proceeding with disintegration of the apical plasma membrane, thereby releasing the cell contents into the ductal lumen (Fig. 5a) and thus establishing a continuity between the ductal lumen and the lumen of the 'fistula' of the principal cell (Fig. 5b,c). The cells affected were individual cells (Fig. 5a,b) or small groups of cells (Fig. 5c,d). Several of the principal cells undergoing this change possessed two nuclei (Fig. 5a,b) and so a large number of released nuclei were seen in the lumen and occasionally formed an occluding density at a location removed from their point of release (Fig. 5e). These nuclei could be clearly distinguished from the nuclei of cells arriving at the epididymis from the testis (Figs 2c and 5d). Mononucleate leucocytes appeared to be attracted towards the sites where the apical plasma membrane of the principal cells opened up (Fig. 6a). Spermatozoa were seen in the fistula of the principal cells (Figs 5d and 7) and even in the basal cytoplasm close to the basal membrane (Fig. 7). Whenever the principal cells opened up to form the fistula, invariably a basal cell was observed at the base of the fistula (Figs 5a-d and 7). In a few sections, the fistula was closed from the ductal lumen owing to neighbouring intact principal cells bending over the vacuole (Fig. 7). When the lumen of such fistulas formed into an intercellular vacuole, an underlying basal cell presumably developed into a PVEC and enveloped the disintegrating principal cells with its contents and the vacuole, which explains the presence of spermatozoa and debris inside the vacuole of such PVECs (Fig. 8a). Fistula formation was not an isolated occurrence since it was found in different sections of the convoluted epididymal duct within the same field (Fig. 8c).

\section{Size and course of the vacuole of the PVEC}

An attempt was made to analyse critically the course of the vacuole inside the epithelium by screening in both directions the serial sections of the epididymis from the midpoint of a few fairly large vacuoles. Such vacuoles were found to extend through $18 \pm 9$ paraffin wax sections, each at $5 \mu \mathrm{m}$ thickness, indicating that the vacuoles extended over a length of $90 \pm 45 \mu \mathrm{m}$ and that the diameter of the largest vacuole was $90 \mu \mathrm{m}$. In most instances, the vacuoles appeared to divide (Fig. 8b). Smaller vacuoles were also seen to merge into larger ones. A proposed sequence of events towards the establishment of the PVEC is indicated (Fig. 9).

\section{Discussion}

The major observations resulting from the present study are that: (1) treatment of mice with AFB1 results in the development of small or large vacuoles in the epithelial lining of all segments of the epididymis. (2) Such vacuoles are enclosed in large pale epithelial cells, which are quite different in organization from the other epididymal epithelial cell types. (3) The lumen of the vacuole contains spermatozoa and debris or an amorphous to dense PASpositive material, or all three materials. (4) Short microvilli extend from the pale epithelial cell into the vacuole. (5) The vacuole appears to arise as a result of the degeneration of a principal cell that leads to fistula formation, during which the content of the ductal lumen and the principal cell fistula merge and spermatozoa from the ductal lumen enter into the fistula. (6) The neighbouring intact principal cells bend over the degenerating principal cell, cutting off its continuity with the ductal lumen. (7) A pale epithelial cell that apparently develops from a basal cell presumably encloses the disintegrating principal cell including the spermatozoa. (8) A pale epithelial cell can have one large or several small vacuoles. The vacuoles in a PVEC may branch and anastomose.

Pale vacuolated epithelial cells have been reported in the epididymal epithelium of normal mice aged 8-16 weeks (Toshimori et al., 1990) and arise as a result of ligation of the 


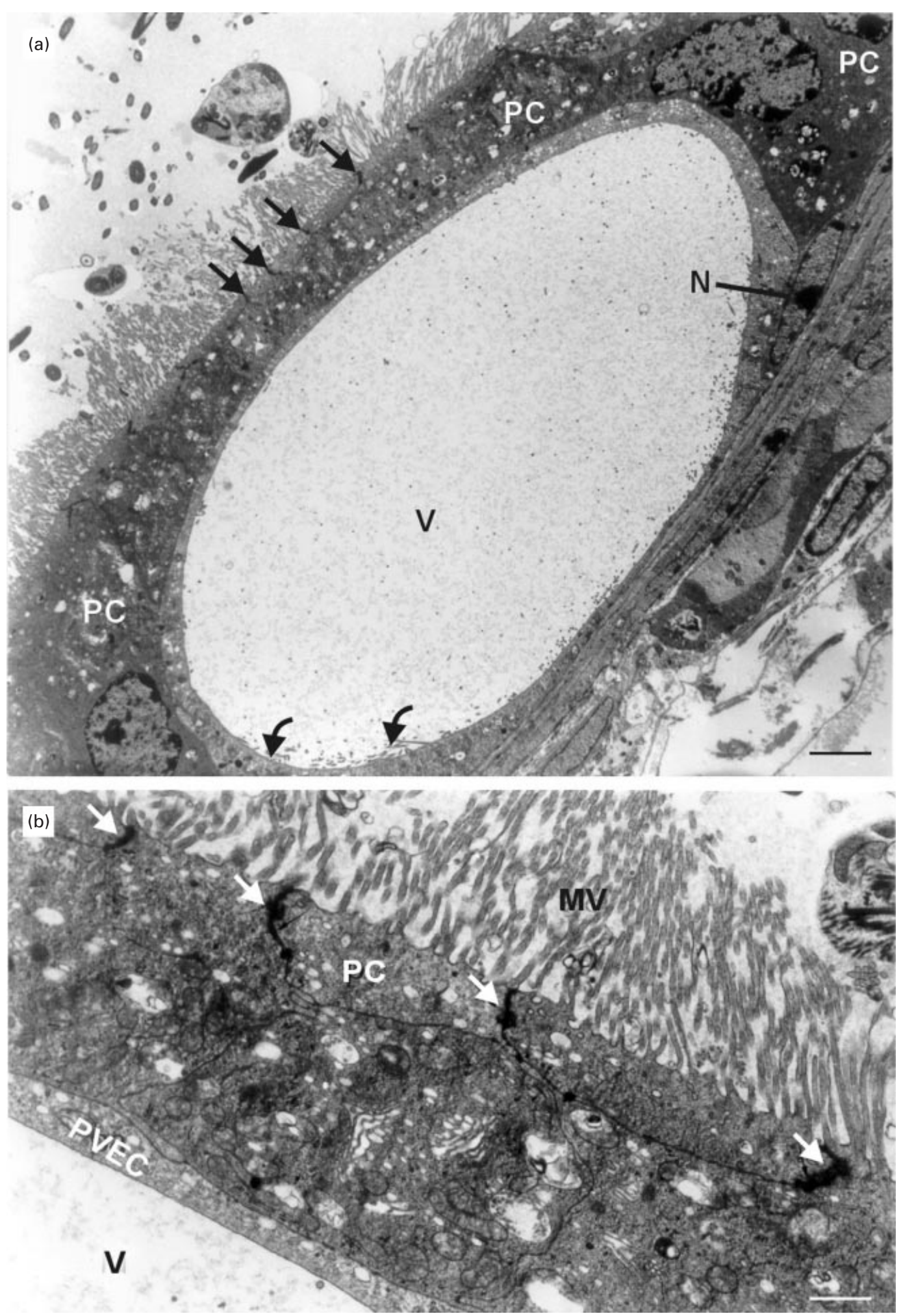

Fig. 4. (a) Transmission electron micrograph showing a pale vacuolated epithelial cell (PVEC), enclosing a vacuole $(V)$ containing an amorphous material, in the epithelium of segment $V$ of the mouse epididymis. Note that principal cells (PC) bend over the PVEC and have developed zonulae occludentes (straight arrows). Curved arrows indicate microvilli. N, nucleus of the PVEC. Scale bar represents $2.1 \mu \mathrm{m}$. (b) Transmission electron micrograph of part of a PVEC and its vacuole $(\mathrm{V})$ with the apical portion of the principal cells (PC) lying on top of the PVEC. Arrows indicate zonulae occludentes formed between principal cells. MV, microvilli of the PCs. Scale bar represents $0.05 \mu \mathrm{m}$. 

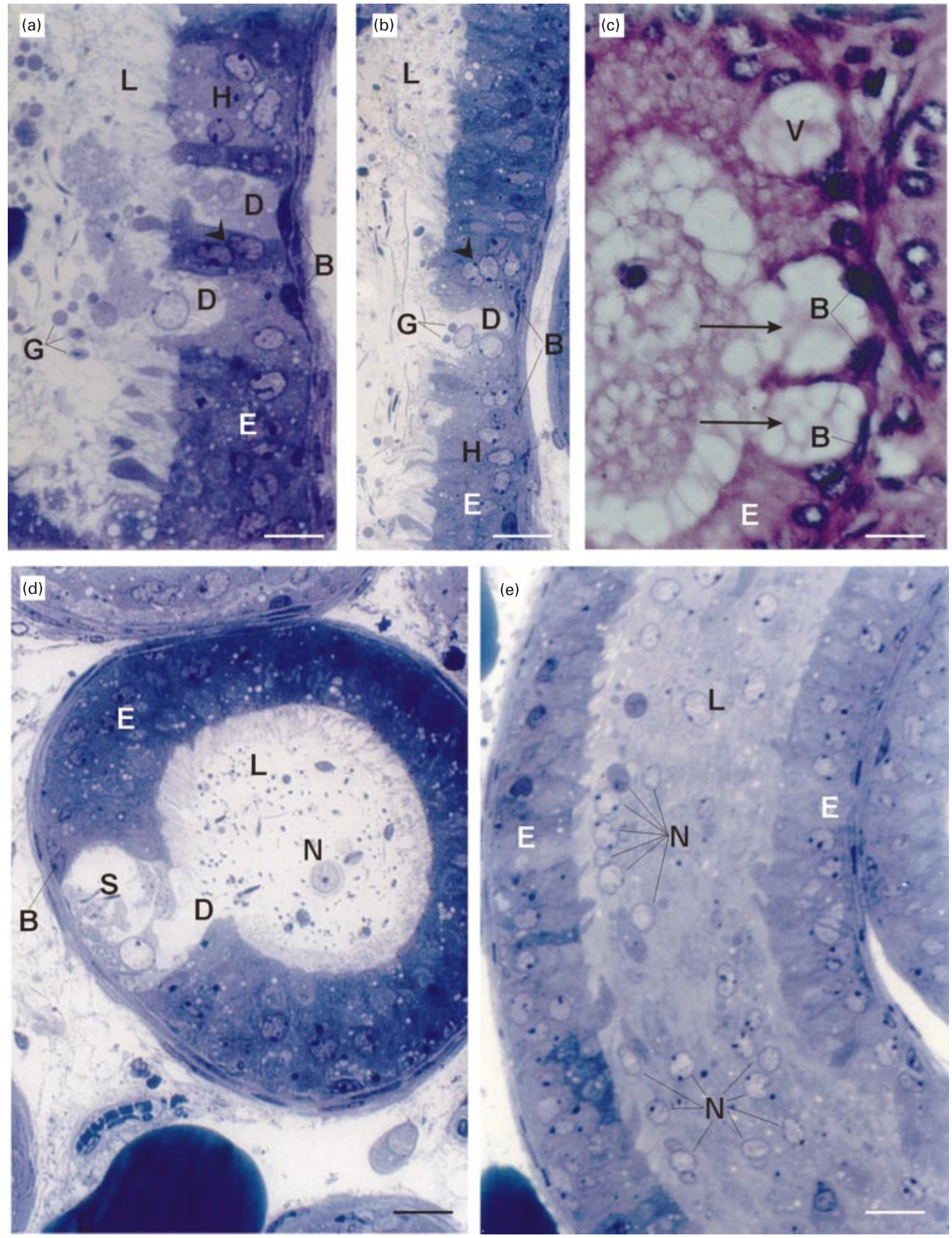

Fig. 5. (a-d) Photomicrographs of the epithelium (E) of the epididymal duct at segment II of mice treated with aflatoxin B1, showing fistula formation in the principal cell. D, degenerating principal cell; $\mathrm{V}$, vacuole; L, ductal lumen; $\mathrm{G}$, glycoprotein granules; $\mathrm{S}$, spermatozoa inside the PC fistula; B, basal cell; N, cell that has arrived at the epididymal duct from the testis; $\mathrm{H}$, hyalination of the PC cytoplasm. Arrows indicate continuity of the ductal lumen with the lumen of the fistula; arrowheads indicate the binucleate nature of the PCs. (e) Section of the epididymal duct at segment IV of a mouse treated with aflatoxin B1, showing occlusion of the ductal lumen (L) by nuclei (N) of PCs from elsewhere discharged after disintegration. Scale bars represent $15 \mu \mathrm{m}$. 

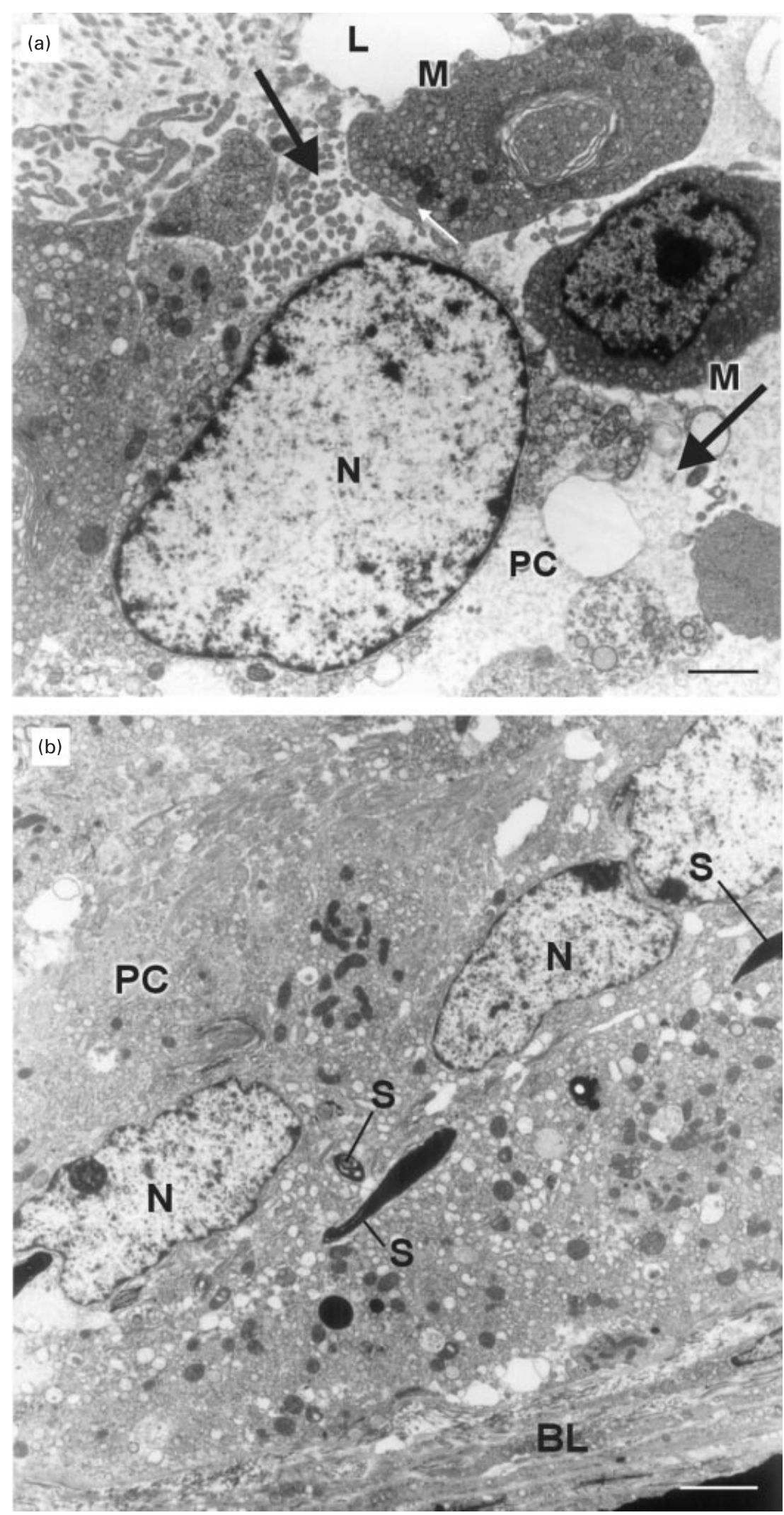

Fig. 6. (a) Transmission electron micrograph showing fistula formation in a principal cell (PC) and attraction of mononuclear leucocytes (M) towards the fistula of the disintegrating PC in mouse epididymis. N, nucleus; L, lumen. Solid arrows indicate continuity between the cytoplasm of the disintegrating PC and the ductal lumen; white arrow indicates the saccular rough endoplasmic reticulum of the leucocyte. Scale bar represents $1.4 \mu \mathrm{m}$. (b) Transmission electron micrograph showing a disintegrating PC that is binucleate (N) with spermatozoa (S) having arrived into the cytoplasm closer to the basal lamina (BL). Scale bar represents $1.8 \mu \mathrm{m}$. 


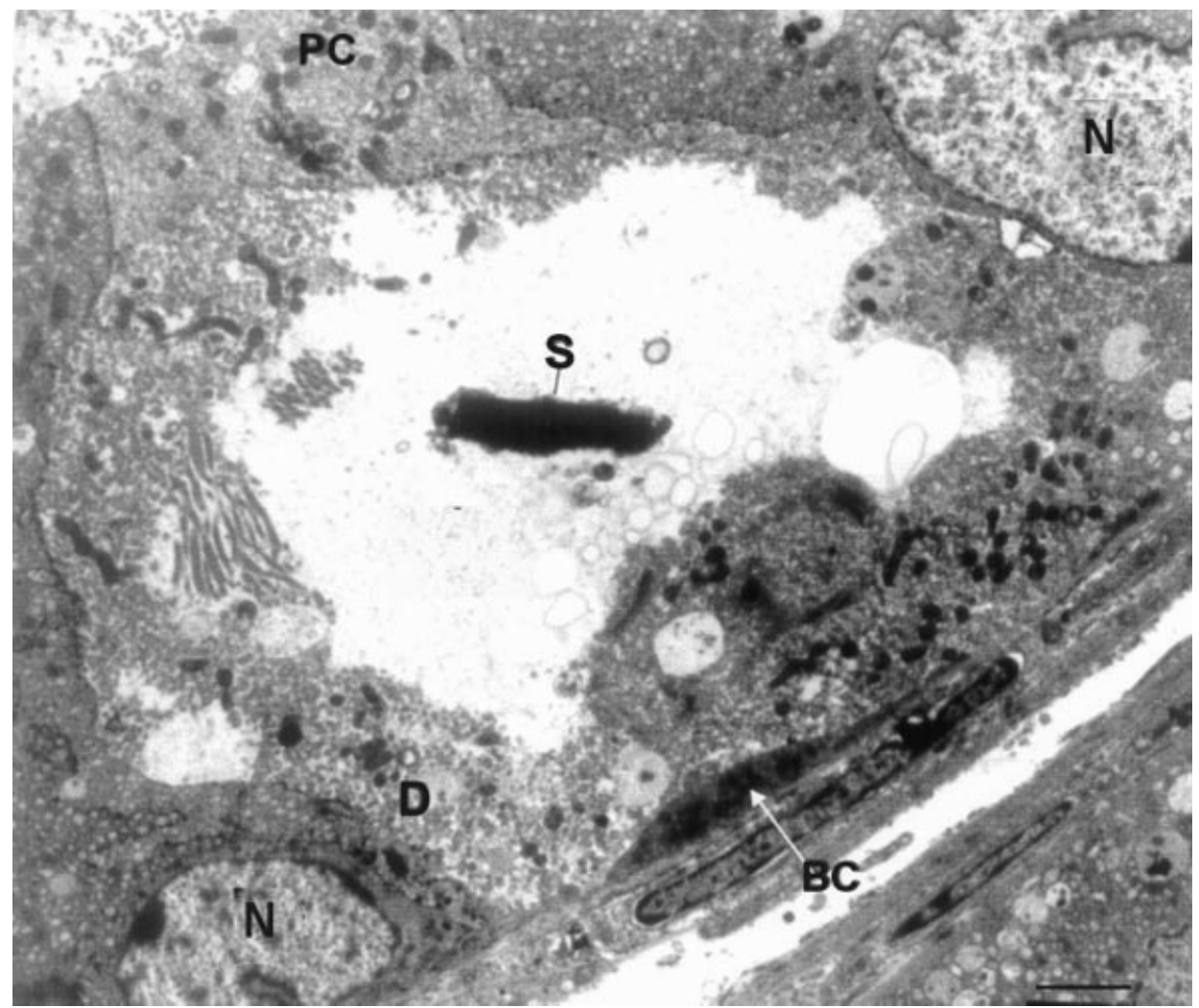

Fig. 7. Transmission electron micrograph showing a disintegrating principal cell (PC) being closed on top by intact PCs in mouse epididymis. A basal cell (BC) is also present, and cell debris (D) and a disintegrating spermatozoon (S) are shown inside the PC fistula. Scale bar represents $1.8 \mu \mathrm{m}$.

epididymal duct at the junction between segments III and IV of 20-30-day-old mice (Abe et al., 1982). Studies on the effect on the epididymis of androgen deprivation in goats (Goyal et al., 1994) and the effect on the epididymis of mice receiving high-dose testosterone implants (Itoh et al., 1999) also show development of such cells. Although the mice used were in the same age group as those used in the study of Toshimori et al. (1990), in the present study, PVECs were not observed in the epididymal epithelium of any of the control mice. Blocking of the PAS-positive material produced by the epithelial cells of segment II from arriving at segment IV (Abe et al., 1982) and hypo-hyperandrogen status (Goyal et al., 1994; Itoh et al., 1999) are the experimental factors so far reported to bring about the development of the PVECs. Although the present study showed PAS-positive secretory granules in the principal cells of the more anterior segments of the epididymis and accumulated in the lumen, and a dense accumulation of spherical cells arriving from the testis at the epididymal duct and apparently blocking the duct, these events did not appear to form the basis for the development of PVECs as no absorption of the ductal luminal PAS-positive material by the principal cells of segment IV of the epididymis was oberved.

Furthermore, according to Abe et al. (1982), PVECs are confined to segment IV of the epididymis and, according to
Toshimori et al. (1990), to segments III, IV and V. Our contention is that the PVECs are produced in all the segments and, hence, their development does not relate to the processing of the PAS-positive material released from the principal cells of segment II (Abe et al., 1982). However, an interpretation relating the development of PVECs with modified androgen status (Goyal et al., 1994; Itoh et al., 1999 ) is relevant in the present context. Although androgen concentrations were not determined in AFB1-treated mice in the present study, AFB1 treatment is known to result in impairment of Leydig cell function and a hypoandrogen status in rats (Egbunike, 1982). In the testis of AFB1-treated mice, pathological changes were found in the Leydig cells (data not shown) and thus it may be inferred that hypoandrogen status is a probable mechanism of action of AFB1 in bringing about the development of PVECs. However, a direct effect of AFB1 or its metabolites on the epididymal epithelium cannot be ruled out.

Abe et al. (1982) used mice of two different age groups, 20-30 days and 90 days, respectively, and found that mice in the younger age group developed more PVECs than those in the older age group, which developed very few or no PVECs. Abe et al. (1982) inferred that after complete differentiation into the definitive segments at sexual maturity, the epididymis does not develop PVECs. On the 

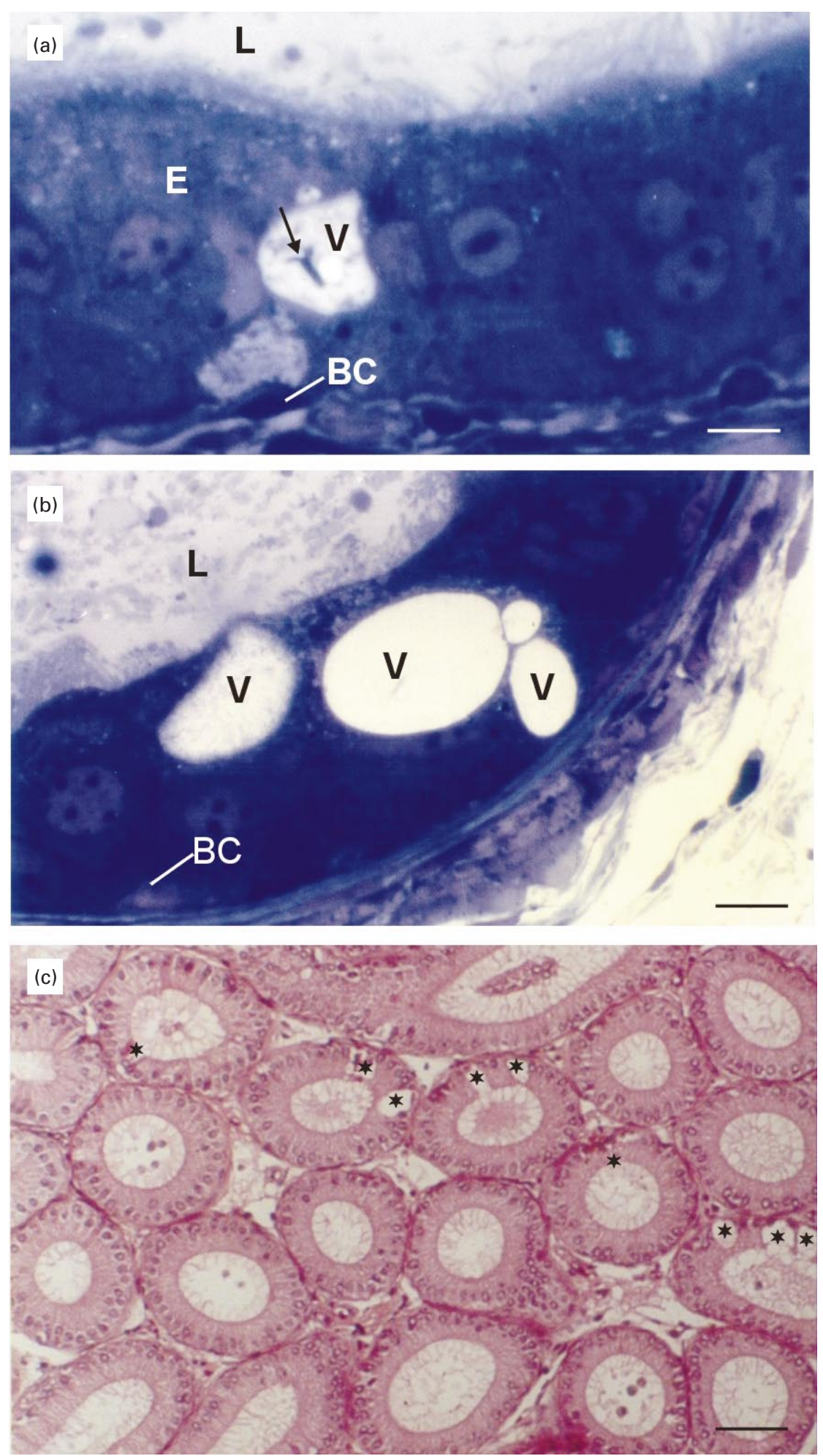

Fig. 8. (a) Photomicrograph of a toluidine blue-O-stained semi-thin section of the epithelium (E) at segment III of the epididymis of a mouse treated with aflatoxin B1, showing a vacuole (V) containing a spermatozoon (arrow) in a pale vacuolated epithelial cell (PVEC). L, ductal lumen; BC, basal cell. (b) Photomicrograph of a toluidine blue-O-stained semi-thin section at segment IV of the epididymal duct of a mouse treated with aflatoxin B1, showing epithelium containing three vacuoles in a PVEC, indicating branching of the vacuole (V). The vacuole on the left may belong to another PVEC. Scale bar represents $15 \mu \mathrm{m}$. (c) Photomicrograph of a haematoxylin and eosin-stained paraffin wax section at segment II of the epididymal duct of a mouse treated with aflatoxin B1, showing the extensive nature of degeneration of the PCs (asterisks). Scale bar represents $65 \mu \mathrm{m}$. 

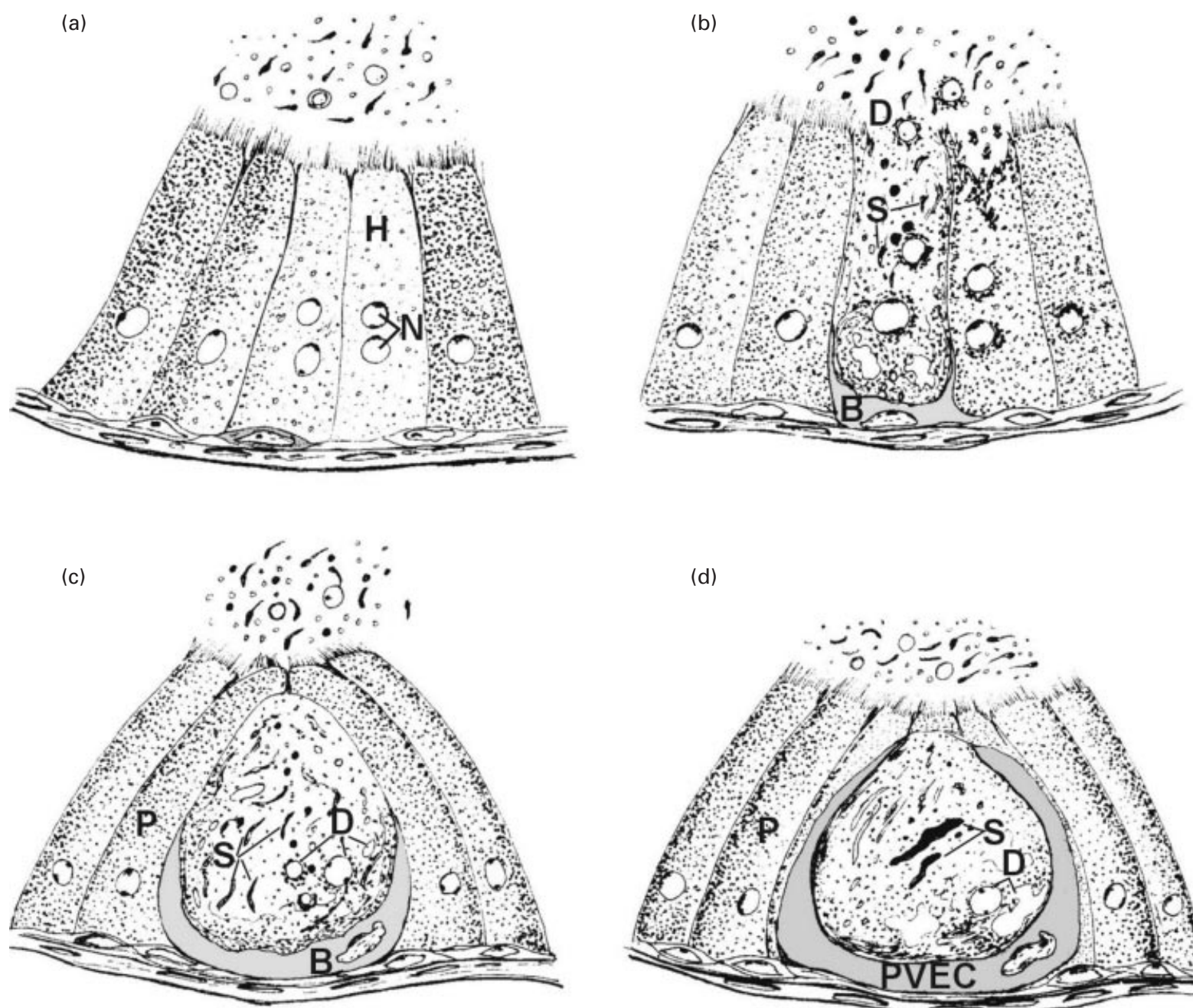

(d)
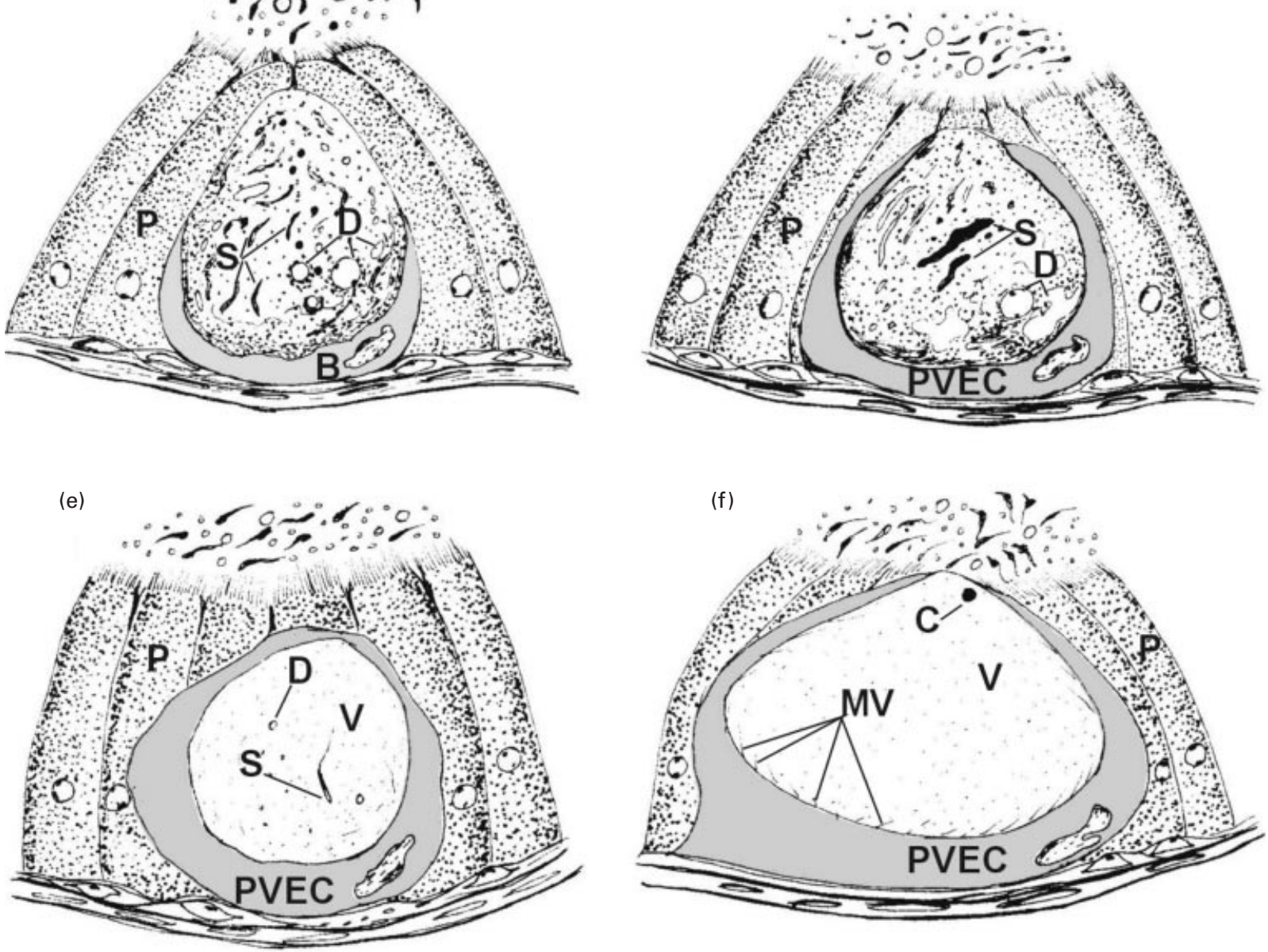

Fig. 9. Schematic model of a tentative sequence in the establishment of a pale vacuolated epithelial cell (PVEC) in segment II in the epididymal epithelium of a mouse treated with aflatoxin B1 (not drawn to scale). (a) Principal cell (PC) becomes binucleate (N) and the cytoplasm becomes hyaline $(\mathrm{H})$. (b) The apical membrane of the hyaline binucleate principal cell disintegrates (D), establishing continuity between the PC cytoplasm and the ductal lumen, whereupon spermatozoa (S) enter into the PC fistula. The basal cell (B) underlying the disintegrating PC has been initiated into development as a PVEC. (c) The neighbouring intact PCs bend over the PC fistula. The basal cell differentiation into PVEC has progressed further. (d) The basal cell is almost fully differentiated into the PVEC; the vacuole contains the spermatozoa (S) and debris (D). (e) The PVEC is fully established, with the vacuole containing a spermatozoon and debris. (f) The spermatozoon and debris of the vacuole (V) of the PVEC have been processed resulting in a dense condensate (C) and a diffuse heterogeneous substance. Microvilli (MV) project into the lumen of the vacuole. 
contrary, the present study in 90-day-old mice indicated that sexual maturity does not prohibit the development of PVECs in the mouse epididymal epithelium.

In general, the structure of the PVECs that develop in the mouse epididymis after AFB1 treatment matches that produced after to ductal ligation (Abe et al., 1982), and PVECs that occur normally (Toshimori et al., 1990). The abundance of endoplasmic reticulum in the stem of the cell (the portion retaining the nucleus) probably reflects the synthetic activity involved in the processing of the contents of the vacuole. The lysosomes and multivesicular bodies in the cytoplasm of the peripheral rim indicate activity of the cell in endocytotic uptake from the interior of the vacuole and further processing. The microvilli reaching into the vacuole reflect a role in absorption. The abundance of mitochondria indicates the involvement of an energy consuming process in the cell. Taken together, the structural organization of PVECs reflects the processing of the content of the vacuoles, absorption and endocytosis of the resultant products and their further intracellular processing. This organization is reflected in the sequence of changes of the luminal content from spermatozoa and debris, through an amorphous material to a highly condensed material.

The preset study shows that, whether it is due to androgen deprivation or a direct toxicity of AFB1 to the epididymis, the starting point for the development of the PVEC is a pathological change in one or more of the principal cells. The content of the vacuole of the PVEC is acted upon as discussed above and results in the formation of cysts, whereupon the size of the vacuole decreases. The content of the vacuole both in the amorphous state and in the cystic form is always PAS-positive. The substance in the PASpositive material is derived from the original content of the lysing principal cell and materials, including spermatozoa, entering into the fistula from the ductal lumen.

Spermatozoa can evoke an autoimmune response if they are extravasated (Nashan et al., 1990; Flickinger et al., 1995, 1998, 1999), as reported in the context of vasectomy and several other experimental treatments (McDonald, 2000; McGinn et al., 2000). Development of fistula in the epididymal epithelium has been shown to allow access for spermatozoa from the ductal lumen into intertubular tissue sites, provoking an autoimmune response of extravasal spermatic granuloma formation (Itoh et al., 1999). Treatment with the fungicide benomyl (Hess and Nakai, 2000) results in the formation of such spermatic granulomas in the ductal lumen itself. Observations of fistula formation in the principal cell in the present study showed spermatozoa accessing the fistula and reaching up to the basal membrane of the cell. Logically, it is only a step further for the spermatozoa to enter the intertubular tissue. It is proposed that the development of PVECs prevents such extravasation of spermatozoa. The arrival of spermatozoa in the principal cell fistula stimulates the underlying basal cell to develop into the PVEC, segregating the spermatozoa and other antigens from the intertubular tissues, which are not otherwise exposed to these antigens. Thus, the PVEC is a protective device preventing the sperm antigens from generating an autoimmune response. This interpretation is substantiated by the observation that, upon disintegration of the apical membrane of the principal cell, mononucleate leucocytes are attracted to this site. A role for the PVEC developing from a basal cell in the surveillance of sperm antigens is supported by the reports of Seiler et al. (1998, 2000) that the basal cells of the mouse epididymis consistently express antigens detected by antibodies against tissue-fixed macrophages (F4/80) and mature macrophages (Mac-1) and play a role in immune defence against sperm autoantigens.

In conclusion, AFB1 treatment of male mice results in pathological changes in the principal cells of the epididymis, irrespective of the segment, whereupon the principal cells form into a fistula, and spermatozoa from the ductal lumen gain access into the fistula. The basal cell in the vicinity of the principal cell apparently develops into a PVEC and encloses the disintegrating principal cell, including the spermatozoa that have entered it, to prevent an autoimmune response to sperm antigens. Presumably, the PVEC acts upon the material enveloped, through digestion in the vacuole, followed by endocytotic uptake and absorption, and lysosomal activity. Hence, it is proposed that the PVEC develops from the basal cell as a protective device against the autoimmune response to spermatozoa in the context of pathological changes in the principal cells.

The ultracut and TEM facility of Wellcome Trust Research Laboratory, Christian Medical College and Hospital, Vellore, India is heartily acknowledged. The work was carried out with financial assistance from the University Grants Commission (UGC), Government of India to V. F. Agnes and from the Department of Science and Technology (DST), Government of India, to M. A. Akbarsha.

\section{References}

Abe K, Takano H and Ito T (1982) Appearance of peculiar epithelial cells in the epididymal duct of the mouse ligated epididymis Biology of Reproduction 26 501-509

Akbarsha MA and Averal HI (1996) Epididymis as target organ for the toxic effect of vincristine: light microscopic changes in the epididymal epithelial cell types Biomedical Letters 54 133-146

Akbarsha MA and Averal HI (1998) Male reproductive toxicity of vincristine: ultrastructural changes in the epididymal principal cell Biomedical Letters 57 159-169

Akbarsha MA and Averal HI (1999a) Epididymis as a target for the toxic manifestation of vincristine: ultrastructural changes in the narrow cell Biomedical Letters 59 113-120

Akbarsha MA and Averal HI (1999b) Epididymis as a target for the toxic manifestation of vincristine: ultrastructural changes in the clear cell Biomedical Letters 59 149-159

Akbarsha MA and Sivasamy P (1998) Reproductive toxicity of phosphamidon: histopathological changes in the epididymis Indian Journal of Experimental Biology 36 34-38

Akbarsha MA, Averal HI, Girija R, Anandhi S and Faridha Banu A (2000) Male reproductive toxicity of vincristine: ultrastructural changes in the epididymal epithelial apical cell Cytobios 102 85-93

Buss P, Caviezel M and Lutz WK (1990) Linear dose-response relationship 
for DNA adducts in rat liver from chronic exposure to aflatoxin B1 Carcinogenesis 11 2133-2135

Chapin RE, Sloane RA and Haseman JK (1997) The relationships among reproductive endpoints in Swiss mice, using the reproductive assessment by continuous breeding database Fundamental and Applied Toxicology 38 129-142

Choy WN (1993) A review of the dose-response induction of DNA adducts by aflatoxin B1 and its implications to quantitative cancer-risk assessment Mutation Research 296 181-198

Cooper TG (1995) Role of the epididymis in mediating changes in the male gamete during maturation Advances in Experimental Medicine and Biology 377 87-101

Cooper TG (1998) Interactions between epididymal secretions and spermatozoa Journal of Reproduction and Fertility Supplement $\mathbf{5 3}$ 119-136

Egbunike GN (1982) Steroidogenic and spermatogenic potentials of the male rat after acute treatment with aflatoxin B1 Andrologia 14 440-446

Egbunike GN (1985) Sperm maturation and storage in the male rat after acute treatment with aflatoxin B1 Andrologia 17 379-382

Egbunike GN, Emerole GO, Aire TA and Ikegwuonu FI (1980) Sperm production rates, sperm physiology and fertility in rats chronically treated with sublethal doses of aflatoxin B1 Andrologia 12 467-475

Fink-Gremmels J (1999) Mycotoxins: their implications for human and animal health Veterinary Quarterly 21 115-120

Flickinger CJ, Howards SS and Herr JC (1995) Effects of vasectomy on the epididymis Microscopy Research and Technique 30 82-100

Flickinger CJ, Baran ML, Howards SS and Herr JC (1998) Epididymal obstruction during development results in antisperm autoantibodies at puberty in rats Journal of Andrology 19 136-144

Flickinger CJ, Bush LA, Williams MV, Naaby-Hansen S, Howards SS and Herr JC (1999) Post-obstruction rat sperm autoantigens identified by two-dimensional gel electrophoresis and Western blotting Journal of Reproduction and Immunology 43 35-53

Goyal HO, Hutto V and Maloney MA (1994) Effects of androgen deprivation in the goat epididymis Acta Anatomica 150 127-135

Hafez AH, Megalla SE and Mahmed AA (1982) Aflatoxin and aflatoxicosis: effect of dietary aflatoxin on the morphology of buffalo bull spermatozoa Mycopathologia 77 141-144

Hess RA (1998) Effects of environmental toxicants on the efferent ducts, epididymis and fertility Journal of Reproduction and Fertility Supplement 53 247-259

Hess RA and Moore DJ (1993) Histological methods for evaluation of the testis. In Methods in Toxicology Vol. 3A pp 52-85 Eds E Chapin and J Heindel. Academic Press, New York

Hess RA and Nakai M (2000) Histopathology of the male reproductive system induced by the fungicide benomyl Histology and Histopathology 15 207-222

Hinton BT and Palladino MA (1995) Epididymal epithelium: its contribution to the formation of a luminal fluid microenvironment Microscopy Research and Technique 30 67-81

Itoh M, Miyamoto K, Satriotomo I and Takeuchi Y (1999) Spermatic granulomata are experimentally induced in epididymides of mice receiving high-dose testosterone implants: a light-microscopical study Journal of Andrology 20 551-558

Karnovsky MJ (1965) A formaldehyde-glutaraldehyde fixative of high osmolarity for use in electron microscopy Journal of Cell Biology 27 $137 \mathrm{~A}$

Kelce WR (1997) The Leydig cell as a target for toxicants. In Comprehensive Toxicology Vol. 10: Reproductive and Endocrine Toxicology pp 113-121 Eds K Boekelheide et al. Cambridge University Press, Cambridge
Klein PJ, Buckner R, Kelly J and Coulombe RA (2000) Biochemical basis for the extreme sensitivity of turkeys to aflatoxin B1 Toxicology and Applied Pharmacology 165 45-52

Klinefelter GR (1997) The epididymis as a target for toxicants. In Comprehensive Toxicology Vol. 10: Reproductive and Endocrine Toxicology pp 151-161 Eds K Boekelheide et al. Cambridge University Press, Cambridge

McDonald SW (2000) Cellular responses to vasectomy International Review of Cytology 199 295-339

McGinn JS, Sim I, Bennett NK and McDonald SW (2000) Observations on multiple sperm granulomas in the rat epididymis following vasectomy Clinical Anatomy 13 185-194

Massey TE, Stewart RK, Daniels J and Liu L (1995) Biochemical and molecular aspects of mammalian susceptibility to aflatoxin B1 carcinogenicity Proceedings of the Society for Experimental Biology and Medicine 208 231-237

Nashan D, Cooper TG, Knuth UA, Schubeus P, Sorg C and Nieschlag E (1990) Presence and distribution of leucocyte subsets in the murine epididymis after vasectomy International Journal of Andrology 13 39-49

Oliveira CA, Kobashigawa E, Reis TA, Mestieri L, Albuquerque R and Correa B (2000) Aflatoxin B1 residues in eggs of laying hens fed a diet containing different levels of the mycotoxin Food Additives and Contaminants 17 459-462

Pier AC (1992) Major biological consequences of aflatoxicosis in animal production Journal of Animal Science 70 3964-3967

Richburg JH (2000) The relevance of spontaneous and chemically-induced alterations in testicular germ cell apoptosis to toxicology Toxicology Letters 15 79-86

Robaire B and Viger RS (1995) Regulation of epididymal epithelial cell functions Biology of Reproduction 52 226-236

Seiler P, Wenzel I, Wagenfeld A, Yeung $\mathbf{C H}$, Nieschlag E and Cooper TG (1998) The appearance of basal cells in the developing murine epididymis and their temporal expression of macrophage antigens International Journal of Andrology 21 217-226

Seiler P, Cooper TG and Nieschlag E (2000) Sperm number and condition affect the number of basal cells and their expression of macrophage antigen in the murine epididymis International Journal of Andrology 23 65-76

Sharpe RM (1992) Are environmental chemicals a threat to male fertility? Chemistry and Industry 3 88-94

Sharpe RM (1993) Declining sperm counts in men: is there an endocrine cause? Journal of Endocrinology 136 357-360

Sotomayor RE, Sahu S, Washington M, Hinton DM and Chou M (1999) Temporal patterns of DNA adduct formation and glutathione Stransferase activity in the testes of rats fed with aflatoxin B1: a comparison with patterns in the liver Environmental and Molecular Mutagenesis 33 293-302

Toshimori K, Araki S and Oura C (1990) Epithelial cells with vacuoles containing 54000 Dalton sialoglycoprotein in the mouse epididymal duct Archives of Histology and Cytology 53 333-338

Trasler JM, Hermo L and Robaire B (1988) Morphological changes in the testis and epididymis of rats treated with cyclophosphamide: a quantitative approach Biology of Reproduction 38 463-479

Wang JS and Groopman JD (1999) DNA damage by mycotoxins Mutation Research 424 167-181

Wogan GN (1999) Aflatoxin as a human carcinogen Hepatology 30 $573-575$

Received 9 February 2001

First decision 21 March 2001.

Accepted 5 June 2001. 